\section{Den Rücken heilen}

- Patienten, die chronisch von Rückenschmerzen geplagt sind, fragen ihre Ärzte oft nach Ratschlägen, was sie selbst tun können, um ihr Leid zu lindern. Das neu bei Irisiana erschienene Buch von Dr. med. Dominik Irnich „Den Rücken heilen“ ist eine gute Empfehlung für wissbegierige Patienten. Die Stiftung Gesundheit hat das Buch zertifiziert.

Der Autor ist Oberarzt an der Klinik für Anästhesiologie und Leiter der Interdisziplinären Schmerzambulanz am Klinikum der Ludwig-Maximilians-Universität München. Am WHO Collaborating Center for TCM in Peking wurde er in Akupunktur und Traditioneller Chinesischer Medizin ausgebildet und leitet inzwischen das Wissenschaftszentrum der Deutschen Ärztegesellschaft für Akupunktur. Seine Forschung zum Thema Schmerz, Naturheilkunde und Akupunktur wurden mit nationalen und internationalen Preisen ausgezeichnet.

Irnich gibt einen ausführlichen Überblick möglicher organischer wie auch psychosozialer Ursachen für den Schmerz und beschreibt, wie aus akuten Beschwerden chronische Schmerzen werden können. Ausführliche Kapitel sind den therapeutischen Möglichkeiten gewidmet. Erläutert werden der richtige Umgang mit Schmerzmitteln, die Physiotherapie, die physikalische Behandlung und die Möglichkeiten komplementärer und naturheilkundlicher Verfahren.

Der Ratgeber zeigt Wege auf, wie Patienten selbstverantwortlich mit ihren Rückenschmerzen umgehen können. Dabei berücksichtigt der Autor bewegungstherapeutische Maßnahmen, aber auch die psychologisch-sozialen Gründe der Schmerzen. Neben den theoretischen Ansätzen enthält das Buch zahlreiche praktische Tipps für Rückenübungen, die einfach, prägnant und auch grafisch dargestellt werden.

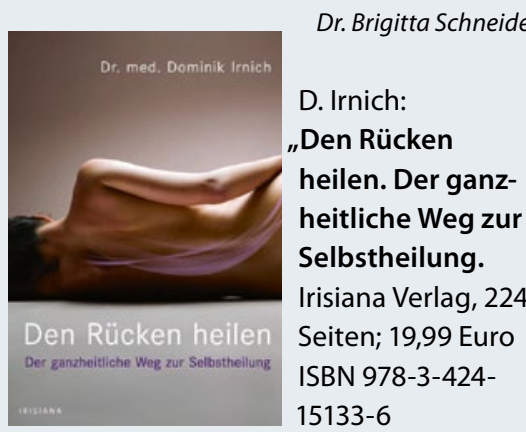

scheidender Bedeutung. Dies zeigt sich bereits dadurch, dass eine ACT auf dem Knochenboden einer erfolglos durchgeführten Mikrofrakturierung signifikant schlechtere Ergebnisse liefert als eine primäre auf einem gesunden Knochen durchgeführte ACT. Ursache für dieses Phänomen sind die oben beschriebenen intraläsionalen Osteophyten, die bei der Mikrofrakturierung entstehen und bei der Präparation des Defektbettes abgetragen werden müssen. In der Folge kommt es zu kleinen Mikroblutungen, die sich mit der Knorpelsuspension vermischen und zu einem minderwertigerem Regenerat führen (Tabelle).

Weiterhin spielt ein optimales Milieu der Synovialflüssigkeit, von der die Ernährung des Knorpels durch Diffusion ausgeht, für das Remodelling des Gewebes eine wichtige Rolle. Deshalb führen degenerative Schäden mit langer Symptomdauer und katabolem Gelenkstoffwechsel eher zu schlechteren Ergebnissen als frische traumatische Knorpelschäden mit physiologischem Gelenkmilieu.

Ferner ist die Mobilisation - zunächst mit einer passiven Bewegungsschiene und später mit einem stufenweisen Belastungsaufbau - bei stabilem Kniegelenk für das Langzeitergebnis von herausragender Bedeutung. Auf eine sechswöchige Teilbelastungsphase folgt die Phase der Belastungssteigerung, sodass je nach Defekt die Vollbelastung nach acht bis zwölf Wochen postoperativ erreicht ist. Low-Impact-Sportarten sollten erst nach vier bis sechs Monaten begonnen werden.

Das selbstständige Training des Patienten spielt unabhängig von der Physiotherapie vor allem in der späten postoperativen Phase für eine optimale Reifung des Gewebes eine ganz entscheidende Rolle. Jeder Patient sollte dabei darüber aufgeklärt werden, dass die Differenzierung des Gewebes und damit der Hyalinisierungsprozess nicht nur Monate, sondern zwei bis drei Jahre in Anspruch nimmt. Dies bedeutet nicht, dass über diesen Zeitraum kein Sport möglich ist, sondern dass erst durch ein gezieltes kontinuierliches Training die beste Stabilität des Gewebes für ein optimales Langzeitergebnis erzielt werden kann.
Fazit

Jeder Knorpelschaden eines Patienten ist anders und benötigt eine exakte Analyse der Ursachen. Die Therapie darf sich dabei nicht auf den Knorpelschaden alleine beschränken, sondern muss immer die Biomechanik des Gelenks mit allen Risikofaktoren wie Bandinstabilität und Achsenfehlstellungen mit berücksichtigen. Ein optimales Ergebnis wird deshalb nur durch ein perfektes Zusammenspiel von sorgfältiger Operation, der Auswahl des richtigen Operationsverfahrens, des individuell auf den Patienten abgestimmten Nachbehandlungsprogramms sowie der Compliance des Patienten erzielt.

Literatur unter www.springermedizin.de/ orthopaedie-und-rheuma

PD Dr. med. habil.

Peter Cornelius Kreuz

Klinik und Poliklinik für Orthopädie

Universitätsmedizin Rostock

Doberanerstr. 142

18057 Rostock

E-Mail: peter.kreuz@uni-rostock.de

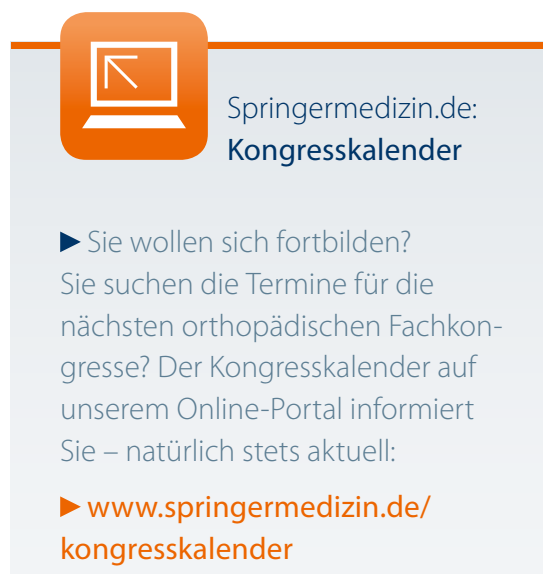

